\title{
High-accuracy differential image motion monitor measurements for the Thirty Meter Telescope site testing program
}

\author{
Lianqi Wang, ${ }^{1, \star}$ Matthias Schöck, ${ }^{2}$ Gary Chanan, ${ }^{1}$ Warren Skidmore,${ }^{2}$ Robert Blum, ${ }^{3}$ Edison Bustos ${ }^{4}$, \\ Sebastian Els, ${ }^{4}$ Reed Riddle, ${ }^{2}$ Juan Seguel, ${ }^{4}$ Tony Travouillon, ${ }^{2}$ Joselino Vasquez, ${ }^{4}$ \\ David Walker, ${ }^{4}$ and Paul Gillett ${ }^{2}$ \\ ${ }^{1}$ Department of Physics and Astronomy, University of California, Irvine, Irvine, California 92697, USA \\ 2Thirty Meter Telescope Project, Pasadena, California 91107, USA \\ ${ }^{3}$ National Optical Astronomy Observatory, Tucson, Arizona 85719, USA \\ ${ }^{4}$ Cerro Tololo Inter-American Observatory, La Serena, Chile \\ *Corresponding author: lianqiw@tmt.org \\ Received 8 March 2007; revised 27 June 2007; accepted 5 July 2007; \\ posted 17 July 2007 (Doc. ID 80819); published 31 August 2007
}

\begin{abstract}
Differential image motion monitors (DIMMs) have become the industry standard for astronomical site characterization. The calibration of DIMMs is generally considered to be routine, but we show that particular care must be paid to this issue if high-accuracy measurements are to be achieved. In a side by side comparison of several DIMMs, we demonstrate that with proper care we can achieve an agreement between the seeing measurements of two DIMMS operating under the same conditions to better than \pm 0.02 arc sec. (C) 2007 Optical Society of America

OCIS codes: $\quad 010.7350,010.1330,010.1080$.
\end{abstract}

\section{Introduction}

Differential image motion monitors (DIMMs) measure the seeing due to atmospheric turbulence by determining star image motion in two small (5-10 cm diameter) pupil plane apertures separated by a distance a few times the aperture size $[1,2]$. A DIMM is a robust instrument requiring only a small telescope (aperture diameters of less than $40 \mathrm{~cm}$ ), a few additional optical elements, and an inexpensive CCD. Furthermore, apart from the pixel scale of the detector, a quantity that is easily measured, the DIMM seeing results depend only weakly on a small number of other parameters, such as the aperture diameters and separation, noise characteristics of the detector, and the exposure time. DIMMs have therefore become the instruments most commonly used for measuring the seeing at astronomical telescope sites.

0003-6935/07/256460-09\$15.00/0

(C) 2007 Optical Society of America
The Thirty Meter Telescope (TMT) project is currently characterizing candidate sites using six identical sets of equipment, including six DIMM systems. To arrive at an informed site decision, it is essential that the reproducibility of measurements taken by each instrument is ensured, even if the instruments are operating at different sites under potentially significantly different conditions. TMT has therefore spent a large effort on calibrating and comparing instruments and determining the factors, both instrumental and environmental, that might bias the results. The results of our DIMM seeing measurement comparison campaign are presented in this paper. We found that the optical quality of the telescope-DIMM system has a larger effect on the seeing measurements than we had previously assumed, at least if an agreement between two instruments of better than 0.1 arc sec is desired.

\section{Experimental Setup}

The TMT seeing monitoring system is mounted on a $35 \mathrm{~cm}$ telescope custom-made by Teleskoptechnik 
Halfmann. For the most part, the DIMM functions as most previously existing DIMMs, by measuring the differential image motion of two stellar images (of the same star) produced by separating the light from two subapertures of the telescope pupil [1]. For the TMT DIMMs, these subapertures are circular, $10 \mathrm{~cm}$ in diameter with their centers separated by $25 \mathrm{~cm}$. Unlike most previously existing instruments, however, the division of the pupil into subapertures and the separation of the two star images of these subapertures are achieved not by a mask in the entrance pupil, but in a reimaged pupil plane behind the telescope optics using tilted mirrors. There, a second set of mirrors also separates the light of another part of the pupil and sends it to the photomultipliers of a multiaperture scintillation sensor (MASS) turbulence profiler [3]. Thus, MASS and DIMM are combined in a single instrument. Only the DIMM part of the MASS-DIMM will be described here.

The other significant difference from most previously built DIMMs is the data acquisition method. To obtain a continuous time series of image motion measurements, data are taken in drift-scanning and binning mode using a Santa Barbara Instrument Group (SBIG) ST-7 CCD. The two star images are projected onto a predefined area of the CCD. After a short exposure time, $T_{e}$, the entire CCD image is shifted by $N$ rows perpendicular to the direction separating the two star images. At the edge of the CCD, the last $N$ rows are binned into a one-dimensional (1D) image and then read out. This process repeats continuously for the total acquisition time, $T_{a}$, during which the shutter is never closed. Typical values for the TMT DIMM are $T_{e}=6 \mathrm{~ms}, N=20-40$, and $T_{a}=36 \mathrm{~s}$. The seeing value is then calculated from such a data set using the standard equations for longitudinal differential image motion [Eq. (8) in [2]].

This method has the advantage of acquiring a higher number of image motion measurements than DIMMs using frame-buffered CCDs that acquire an exposure every few hundred milliseconds (typically, the TMT DIMMs record 6000 measurements in an acquisition compared to several hundred for frame transfer DIMMs). Because a continuous time series exists, we can also bin consecutive rows of data to obtain images of longer exposure times, which are then used to extrapolate the seeing measurements to zero exposure time. In practice, we perform this extrapolation using both a "two-point" method [2] with exposure times $T_{e}$ and $2 T_{e}$, and an exponential fit using six different exposure times, from $T_{e}$ to $6 T_{e}$. It should furthermore be possible to use the continuous time series to obtain at least qualitative estimates of the turbulence time constant. This is not yet implemented and will be investigated in the future. The disadvantage of this method is, of course, that the image motion perpendicular to the line separating the two star images, the transverse image motion, is lost. As longitudinal and transverse image motion independently provide the same seeing measurements if sufficient care is taken, the advantages outweigh this loss. Refer to Table 1 for a detailed list of
Table 1. Detailed Parameters of the TMT DIMM Systems During the Campaign and Actual Site Testing

\begin{tabular}{|c|c|}
\hline Parameters & Value \\
\hline Pupil plane subaperture diameter & $98 \mathrm{~mm}$ \\
\hline $\begin{array}{l}\text { Pupil plane subaperture separation } \\
\text { of centers }\end{array}$ & $240 \mathrm{~mm}$ \\
\hline Pixel scale & $0.78 \mathrm{arc} \mathrm{sec} / \mathrm{pixel}^{a}$ \\
\hline CCD pixel size & $9 \mu \mathrm{m}$ \\
\hline Telescope focal length & $2.8 \mathrm{~m}$ \\
\hline Window height & 40 pixels $^{b}$ \\
\hline Window width & 100 pixels \\
\hline Typical image separation & 35(T2), 65(T3) pixels \\
\hline Exposure time & $6.1 \mathrm{~ms}^{c}$ \\
\hline Number of exposures & 6000 \\
\hline $\begin{array}{l}\text { Wavelength for which seeing is } \\
\text { calculated }\end{array}$ & $500 \mathrm{~nm}$ \\
\hline $\begin{array}{l}\text { Wavelength of peak CCD } \\
\text { sensitivity }\end{array}$ & $625 \mathrm{~nm}$ \\
\hline Radius used in centroiding & 2 pixels $^{d}$ \\
\hline
\end{tabular}

${ }^{a}$ Pixel scale is measured using binary stars.

${ }^{b}$ The stellar images are centered around the middle of the CCD window and are also aligned along the rows of the CCD. The telescope can position the target to within a couple of pixels and there are no significant tracking errors, i.e., the tracking errors are less than a pixel, during an exposure. The window size easily accommodates the two stellar images in both height and width (separation).

${ }^{c}$ We use the same window height at all sites, so exposure time is the same.

${ }^{d}$ The centroiding box is 5 pixels wide including the central pixel. The central pixel is set to the brightest pixel in each stellar image.

the instrumental parameters. It is worth noting that the telescopes used by the TMT site testing systems are open structures, thus they cannot be subject to internal turbulence.

In the following sections, we use the Strehl ratio of the DIMM data as a measure of data quality. To be able to compare our results more easily with DIMMs taking two-dimensional (2D) star images, we estimate the Strehl ratios of the original 2D images from our data rather than using the Strehl ratio of the 1D data as they are taken. We approximate the $2 \mathrm{D}$ star image by a radially symmetric Gaussian,

$$
I_{2 \mathrm{D}}(x, y)=I_{0,2 \mathrm{D}} e^{-\left(x^{2}+y^{2} / 2 \sigma^{2}\right)},
$$

where $I_{0,2 \mathrm{D}}$ is the central intensity of the image and $\sigma$ is the root-mean-square value of the Gaussian in each dimension. Integrating over $y$ gives

$$
I_{1 \mathrm{D}}(x)=\sqrt{2 \pi} \sigma I_{2 \mathrm{D}}(x, 0) .
$$

Thus, the intensity in each pixel of the 1D image is a factor $\sqrt{2 \pi} \sigma$ higher than in the original $2 \mathrm{D}$ image. In particular, we also get the central intensity of the 1D image to be $I_{0,1 \mathrm{D}}=\sqrt{2 \pi} \sigma I_{0,2 \mathrm{D}}$. The equation for the Strehl ratio of the image formed by a circular aperture of diameter $D$ is

$$
S_{2 \mathrm{D}}=\frac{I_{0,2 \mathrm{D}}}{I_{\text {total }}} \frac{4 \lambda^{2}}{\pi D^{2} \delta x^{2}}
$$


where $I_{\text {total }}$ is the total intensity in the image, $\lambda$ is the observation wavelength, and $\delta x$ is the pixel size in radians under the assumption that $\delta x$ is small compared to the size of the Airy disk. Using this, we can estimate the Strehl ratio of the original 2D star images from the 1D time series of DIMM data using

$$
S_{2 \mathrm{D}}=\frac{I_{0,1 \mathrm{D}}}{I_{\text {total }}} \frac{4 \lambda^{2}}{\sqrt{2 \pi} \sigma \pi D^{2} \delta x^{2}} .
$$

In practice, we use the intensity of the brightest pixel for $I_{0,1 \mathrm{D}}$, determine the full width at halfmaximum, $w_{\text {FWHM }}$, of each image with $\sigma=w_{\text {FWHM }} /$ $(2 \sqrt{2 \ln 2})$ for a Gaussian, and average over all images in a DIMM data set. In addition to the approximations that the images are Gaussian and that the pixel size is small in the above sense, $I_{\text {total }}$ is calculated as the sum of intensities in only a few pixels centered around the brightest pixel and might therefore be underestimated, especially for low Strehl ratios (wide images). Thus, the calculated Strehl ratio is an approximate value and can only be used as a guideline when comparing to other DIMMs. It is, however, consistent between all TMT DIMMs, which use identical hardware and software.

\section{Data}

From August to October 2004, two identical MASSDIMM systems were set up at Cerro Tololo InterAmerican Observatory (CTIO). The telescopes were mounted on towers such that the primary mirrors were at equal heights, approximately $7 \mathrm{~m}$ above the ground. The two systems, referred to in the following as T2 and T3, were separated by approximately $5 \mathrm{~m}$ horizontally and were located close to the northern edge of the Cerro Tololo summit plateau, such that the air flow was unperturbed for the prevailing northern wind directions.

Data acquisition was coordinated in that both telescopes always pointed to the same star. However, the start times of individual data acquisitions were not synchronized. We used acquisition times of $36 \mathrm{~s}$ during the campaign. Consecutive acquisitions were approximately $90 \mathrm{~s}$ apart. For the comparisons presented below, the T3 data were interpolated to match the acquisition times of $\mathrm{T} 2$.

\section{Data Quality Control}

At the beginning of the DIMM comparison campaign, the differences between the seeing values measured by the T2 and T3 DIMMs were large and systematic, at times reaching 0.2 arc sec. An example of this is shown in Fig. 1 (top left), where the seeing measured by T2 and T3 during the night of 29 August 2004 is plotted. To reduce scatter, data points are binned in 15 min intervals. Even though the two instruments were separated by only $5 \mathrm{~m}$ horizontally, were at the same elevation above the ground, and were pointing to the same stars, T2 measured consistently worse seeing than T3 over a large range of seeing values.

Through a series of tests we eventually determined that our DIMM seeing measurements are more de- pendent on the optical alignment of the telescopeDIMM system (mostly secondary collimation; defocus can be controlled actively or remotely in the TMT DIMMs) than we had originally expected. It is well known that optical aberrations of a DIMM bias the measured seeing because of scintillation and the effect of higher-order aberrations [4]. Thus, it was not surprising to find the seeing measurements to be dependent on the optical alignment, but the sensitivity of the DIMM measurements to this effect was unexpected. We also found that the Strehl ratios of the DIMM data sets can be used as a reliable measure of this optical quality and that no other method to confirm correct optical alignment is needed. This is also demonstrated in Fig. 1. In the bottom left of the figure, we show the mean Strehl ratios of the T2 and T3 DIMMs during the night of 29 August. T3 had mean Strehl ratios around 0.4, while T2 Strehls were around 0.3 , indicating a better optical alignment of T3. Follow-up investigations showed that T2 was indeed misaligned, albeit at a level that we would have considered not critical before the comparison campaign. After realigning the T2 optics, we found T2 and T3 DIMM measurements to be in much better agreement. An example of this, the night of 19 September, is shown in the top right plot of Fig. 1.

Figure 2 shows how the measured seeing difference depends on the DIMM Strehl ratios for all nights between 13 August and 26 October. The abscissa in this figure is the minimum of the four Strehl ratio values of the two DIMMs (each DIMM having two subapertures). We can see that a systematic difference larger than a few tens of milliarcseconds is introduced when Strehl ratios drop to values around
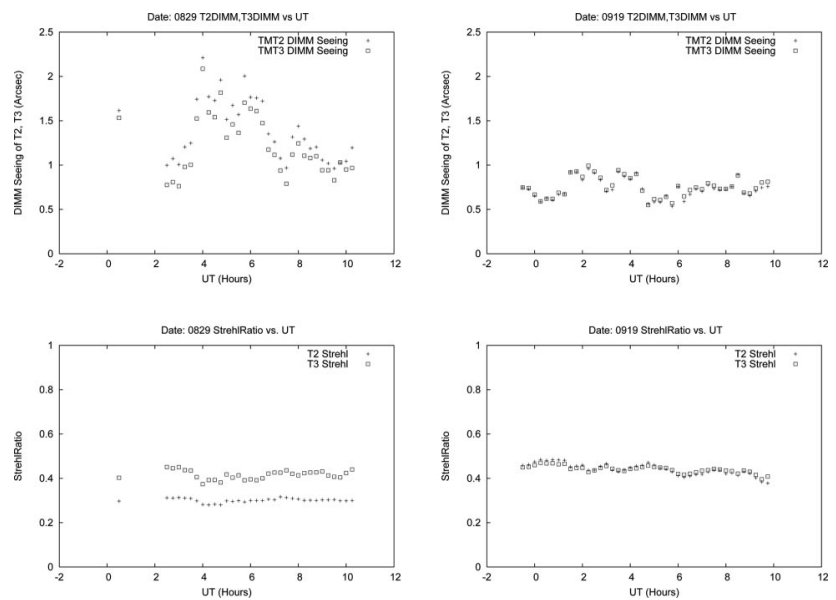

Fig. 1. Examples of DIMM measurements taken during two individual nights (left: 29 August 2004; right: 19 September 2004). Seeing measurements and Strehl ratios are binned in $15 \mathrm{~min}$ intervals in order to reduce scatter. The bottom plots show the average Strehl ratios (mean Strehl ratio of two DIMM images) of the DIMM data. We can see a significant difference between the two DIMMs on 29 August, when the T2 Strehl ratios were low. On 19 September, after the optical alignment of T2 was improved, T2 Strehls were higher and good agreement in seeing measurements was found. 


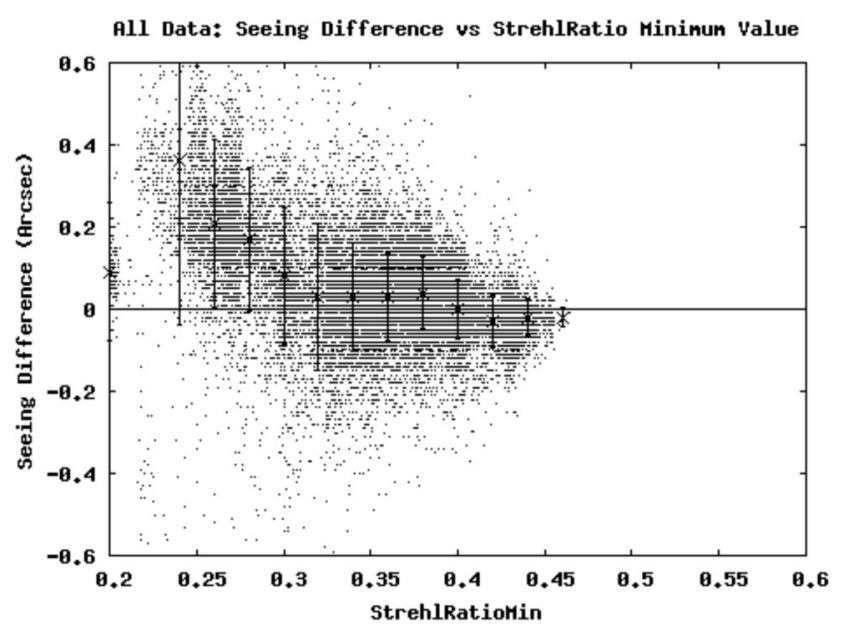

Fig. 2. Plot of the measured seeing difference between T2 and T3 (T2-T3) versus the minimum Strehl ratio for the period from 13 August to 26 October 2004. The error bars are the standard deviation of the seeing difference for respective Strehl ratio bins. As the minimum Strehl ratio decreases, an increasing seeing difference between the two DIMMs is found.

0.35. (This difference is systematic because, throughout our comparison campaign, alignment problems were experienced only for T2.) Figure 2 thus shows that it is important to assure good optical alignment if high-accuracy DIMM measurements are required. The Strehl ratio can be used as metric of the quality of optical alignment.

\section{A. Seeing-Independent Strehl Ratio Limit}

For the TMT DIMMs, we first adopted a Strehl ratio of 0.4 as the limit above which data can without any doubt be considered uncorrupted by telescope and DIMM optical aberrations. This value was selected because Fig. 2 shows that no significant bias is introduced until the Strehl ratios drop to well below 0.4 and because, in medium and good seeing, we can routinely achieve and maintain Strehl ratios larger than 0.4 with our DIMMs. Note that the optimal value of this limit is likely different for other DIMMs, especially those using $2 \mathrm{D}$ images.

\section{B. Seeing-Dependent Strehl Ratio Limit}

The Strehl limit cannot, however, be set as a rigid limit of DIMM data validity because the Strehl ratio also depends on the seeing itself. If we simply were to discard all data with Strehl ratios less than 0.4 we would introduce a bias toward better seeing measurements. This is demonstrated in Fig. 3, which shows the seeing and Strehl ratios for all T2 and T3 data collected at CTIO. During the campaign, T2 experienced three different levels of optical alignment, which can be seen by the appearance of three distinct regions in the left panel of the figure. Each of these regions as well as the data in the right panel (T3) show the expected drop of Strehl ratios as the seeing gets worse, with values frequently dropping below 0.4 even for the well-aligned cases. A seeing-dependent Strehl ratio limit is therefore needed.
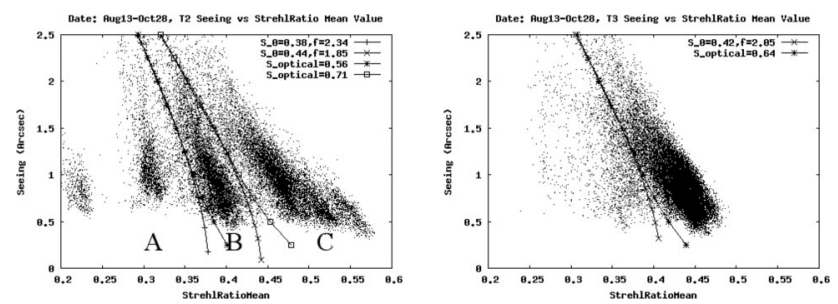

Fig. 3. Seeing values versus mean Strehl ratio for T2 (left panel) and T3 (right panel) from the 2004 Tololo comparison campaign. All data are shown, including periods when the optical alignment was not good. Data points are divided into three discrete regions due to different levels of optical alignment. Region C corresponds to the period of good optical alignment of T2. Also shown are the theoretical and simulated Strehl limit curves for different values of $S_{0}$ and $f$ (theoretical curve) and $S_{\text {optics }}$ (simulated curve). See the legends in the plots for the values used for each curve.

We took two different approaches to develop this seeing-dependent Strehl ratio limit, one using simulations, the second based on theoretical equations. The simulations were done to verify that the behavior observed in the data is indeed caused by the effects described above. The theoretical equations were developed as they are simpler to apply to the large data sets from the TMT candidate sites than simulation results.

Simulations of the DIMM system were done using the DIMM simulation package developed by Tokovinin at CTIO [4]. Each simulation was carried out as follows. Two turbulent layers were generated, one at $0 \mathrm{~km}$ containing $90 \%$ of the total turbulent strength and the other at $10 \mathrm{~km}$. The total turbulence strength, described by $r_{0}$, varied from 0.05 to $0.4 \mathrm{~m}$ for different simulation runs. Star light was propagated through the two atmospheric layers and then transformed by two DIMM subapertures to get star images. The subaperture size and separation, CCD pixel scale, exposure time, etc. were all matched to the TMT DIMM instruments. Various amounts of coma and astigmatism aberrations were included in both subapertures to simulate different optical qualities of the TMT DIMM system. The images were binned into one dimension in the same way as in the TMT drift-scanning and binning mode. Seeing and Strehl ratios were then calculated using the same equations as the TMT code. Finally, the simulated seeing was plotted versus the Strehl ratio for different seeing conditions and results for different levels of optical aberrations. The result is shown in Fig. 4. The numbers in the top right show the respective optical Strehl ratios. Data points from T2 are also shown for comparison. The data regions observed before are well-bounded by the curves.

The theoretical calculation of the Strehl-dependent seeing limit is based on a number of simplifying assumptions.

- The optical quality of the system is assumed to be high. We can therefore use the extended Maréchal approximation, $S=\exp \left(-\sigma^{2}\right)$, where $S$ is the Strehl ratio and $\sigma^{2}$ is the variance of the wavefront phase in 


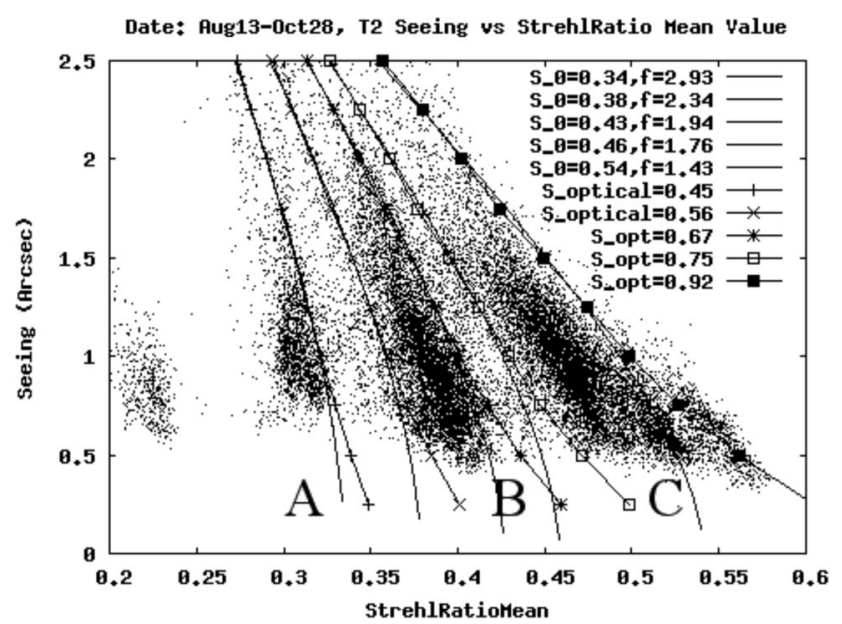

Fig. 4. Five curves with symbols (bending to the right for low seeing values) show seeing versus Strehl ratios for simulated DIMM data with different levels of optical aberration as shown in the legend. The other five curves are the theoretical curves calculated from Eq. (5) that best fit the simulated curves. The respective values of $S_{0}$ and $f$ are also indicated in the legend. Increasing values of $S_{0}$ correspond to shifting the curves to the right. The background points are those of T2 and are the same as in Fig. 3.

square radians. As we generally find Strehl ratios in excess of 0.35 , this approximation is valid.

- Because Strehl ratios are large, we can express the overall Strehl ratio as the product $S=S_{\text {optics }} S_{\text {turbulence. }}$.

- DIMM exposures, being $6 \mathrm{~ms}$ long, are sufficiently short that the tip-tilt component of the wavefront can be considered constant during the exposure. The image width due to turbulence is therefore that caused by a tip-tilt removed wavefront. Thus, the variance of the wavefront phase is given by $\sigma^{2}=$ $0.134\left(d / r_{0}\right)^{5 / 3}$, where $d$ is the diameter of the DIMM subaperture, $r_{0}$ is the Fried parameter with $\epsilon=$ $0.98 \lambda / r_{0}, \epsilon$ is the seeing in radians, and $\lambda$ is the wavelength of light [5]. We use the standard $\lambda=0.5 \mu \mathrm{m}$ for all our calculations.

The seeing-dependent Strehl ratio limit is then given by

$$
S_{\text {limit }}(\epsilon)=S_{0} \exp \left[-\frac{0.134}{f}\left(\frac{\epsilon d}{0.98 \lambda}\right)^{5 / 3}\right]
$$

Here $S_{0}$ is, in principle, the lowest Strehl ratio of the optical system alone for which seeing values can be considered unaffected by optical aberrations. However, as not all the assumptions made in the development of Eq. (4) are satisfied by our DIMMs in all conditions (in particular, the pixel size is not small compared to the Airy disk in good seeing), the Strehl ratio calculated from our data is not strictly the real physical Strehl ratio of the incoming wavefront. When applying Eq. (5) to our data, $S_{0}$ is therefore a parameter related to the Strehl ratio of the optical system, $S_{\text {optics }}$, but not $S_{\text {optics }}$ itself. This is illustrated in the legends of Figs. 3 and 4, where the curves show both the theoretical Strehl limits for different values of $S_{0}$ and the simulation results for different levels of optical aberrations. It can be seen that the optics Strehl ratios and the values of $S_{0}$ follow the same trend, but that they are not identical.

In addition, an empirical factor, $f$, is introduced in Eq. (5) in order to match the theoretical Strehl limit equations with the curves found from the simulations. This factor is larger than unity because the practical calculation of the Strehl ratio uses only a few pixels and thus underestimates $I_{\text {total }}$ in Eq. (4). The calculated Strehl ratio therefore overestimates the real Strehl ratio of the data, in particular in bad seeing. The pairs of $S_{0}$ and $f$ which produce the best fits between simulated and theoretical data are given in the first two columns of Table 2. In Figs. 3 and 4 as well as in the following, we always use these combinations of $S_{0}$ and $f$. Note that these values apply only to the TMT DIMMs (and, strictly speaking, only to the kind of optical aberrations and turbulence that we simulated) and will be different for other DIMMs. We have found, however, that our results depend only weakly on the exact choice of $f$ and that using an approximate value does not bias the seeing statistics.

With these adjustments, Figs. 3 and 4 show that the theoretical curves closely follow the simulation results for the ranges of seeing values and Strehl ratios that occur frequently in practice. Thus, Eq. (5) can be used to distinguish between valid and biased seeing measurements.

As a final note, we point out that the seeing in Eq. (5) is, in principle, the intrinsic atmospheric seeing rather than the measured seeing. However, as we use the equation to define the limiting case for which the optical quality of the system is sufficiently high that it has a negligible effect on the measured seeing, the measured seeing can be used instead.

Table 2. Dependence of Median Seeing and Remaining Number of Data Points on $S_{0}$ and the Corresponding Best-Fitting Factor $f^{a}$

\begin{tabular}{cccc}
\hline$S_{0}$ & $f$ & $N_{\text {points }}$ & Normalized Seeing \\
\hline 0.00 & - & 284186 & 1.011 \\
0.20 & 5.99 & 284151 & 1.011 \\
0.30 & 3.69 & 283454 & 1.011 \\
0.35 & 2.69 & 278859 & 1.006 \\
0.40 & 2.14 & 260398 & 1.000 \\
0.45 & 1.81 & 206423 & 0.992 \\
0.46 & 1.75 & 186466 & 0.990 \\
0.47 & 1.70 & 163598 & 0.986 \\
0.48 & 1.66 & 134610 & 0.978 \\
0.50 & 1.57 & 72719 & 0.957 \\
0.55 & 1.40 & 17333 & 0.891 \\
\hline
\end{tabular}

${ }^{a}$ The third column shows the number of data points remaining after the Strehl limit was applied and the fourth column is the corresponding median seeing [normalized by the seeing for $S_{0}=$ 0.4 ]. It can be seen that the median seeing remains constant to $\sim 2 \%$ until more than half of the data points have been eliminated. See text for more explanations. 
C. Testing the Seeing-Dependent Strehl Ratio Limit with Real Data

As a final step, we investigated the available data for corroborating evidence that the theoretical limit is, indeed, applicable to real data and does not introduce a bias. This is done using the following argument. The optical alignment of the telescope and DIMM is constant over short time scales, but varies somewhat over longer time scales due to gravitational effects, mechanical drifts, and temperature changes. It is, however, entirely independent of the seeing. The measured seeing values, in turn, do not depend on the optical alignment as long as the optics are sufficiently well-aligned. Therefore, the choice of $S_{0}$ (and the corresponding $f$ ) should not bias the seeing statistics as long as it is sufficiently high that optical aberrations do not affect the image quality and sufficiently low that enough data points remain for the statistics to be representative of the conditions.

This argument cannot be tested with the Tololo campaign data, as there are not enough data points available. We can, however, test it with the data from one of the TMT candidate sites, Cerro Tolar. Table 2 shows how the median seeing and the remaining number of data points change when progressively higher limits of $S_{0}$ are used. The third column contains the number of data points remaining after the Strehl limit was applied. The fourth column is the median seeing (normalized by the seeing for $S_{0}=$ 0.4) for these data points. We see that the median seeing remains constant to better than $2 \%$ even if half of the data points are eliminated. The remaining difference might be caused by the Strehl limit, or it might simply be due to a small difference in the turbulence statistics of the site for the two halves of the data set. In either case, it is so small that it is of no concern for our data.

Whether the median seeing increases or descreases with $S_{0}$ depends on the shape of the Strehl limit curve used. If it is flatter than the real limiting curve, more data points with small seeing values will be excluded and more high values included, thus producing a bias toward larger median seeing. If it is steeper, the opposite will occur. As a test, we also selected the data based on the simulated curves. This resulted in changes of the median seeing of similar magnitude to those of Table 2, but with opposite sign. For the conditions encountered in this data set, the shape of the simulated curves are thus slightly too flat, while the theoretical curves are slightly too steep. However, for the values that are of most interest for data quality control of our DIMMs, that is, for $S_{0}$ between 0.35 and 0.4 , the bias is negligible for both methods. We therefore generally use the theoretical curve as it is simpler to apply in practice.

Finally, note that the use of no Strehl limit, $S_{0}$ $=0$, introduces no bias for this data set as great care is taken that the optics of the TMT DIMMs at the candidate sites are always well-aligned.

\section{Focusing}

Once good optical alignment of the telescope-DIMM system is assured, for example, by measuring the Strehl ratios during periods of good seeing, the separation of the two star images on the CCD can also be used as a data quality criterion. This separation, averaged over a DIMM data set, is a sensitive measure of the defocus of the telescope. For DIMM systems mounted on small telescopes, changes in focus position due to temperature variations usually dominate optical aberrations and are often sufficiently large that they bias the seeing measurements. By establishing an acceptable range of focus positions with the Strehl criterion and measuring them through the image separation, data can be accepted or rejected based on their image separation. The separation then also tells the operator whether it is necessary to refocus the telescope. This method has the added advantage that it is independent of seeing and therefore introduces no statistical bias. For the TMT telescopes, operator intervention is generally not necessary, as the thermal expansion of the telescope tube is negligible and the secondary mirror position is actively controlled with accuracies of the order of one micrometer. Thermal expansion as well as mechanical deformation of the DIMM instrument itself dominate the defocus terms, but have been shown to be sufficiently small to have no effect on the seeing measurements. We therefore regularly monitor the image separation, but operator intervention or data rejection based on the image separation is usually not required.

\section{Results}

After setting up the Strehl ratio metric, we compiled all data from the days for which we know both telescope-DIMM systems to have been well-aligned; that is, from 15 September to 5 October 2004. Figure 5 shows a scatterplot of these measurements. The T2-T3 difference is shown versus UT in Fig. 6. The plot versus UT is done here not in order to look for

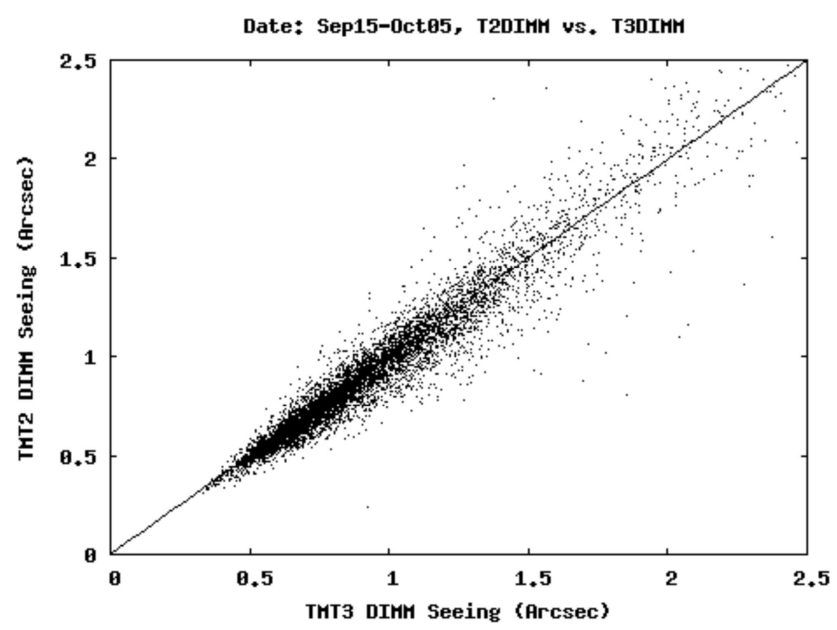

Fig. 5. Scatterplot of the measured seeing values for T2 and T3 for the period between 15 September and 5 October 2004. 


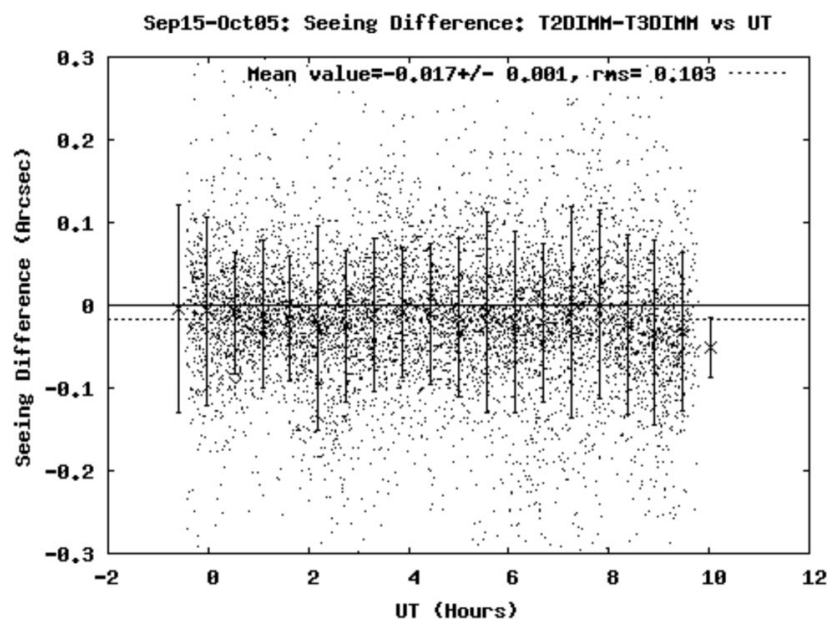

Fig. 6. Difference between the two DIMM measurements (T2-T3) for the period between 15 September and 5 October 2004. The data are folded onto a $24 \mathrm{~h}$ UT day simply to spread out the points. The error bars show the standard deviation of seeing difference in respective UT bins. The scatter is mostly caused by local turbulence effects. The mean systematic difference is $-0.017 \pm 0.001$ arc sec, in which \pm 0.001 arc sec is the standard deviation of the seeing difference.

diurnal trends (which do not seem to exist), but simply to spread out the data points. We can see that the two instruments agree on average very well, the remaining difference being $-0.017 \pm 0.001$ arc sec. There remains a considerable amount of scatter $( \pm 0.1$ arc sec) that is much larger than the systematic difference. This scatter is primarily caused by differences in the turbulence seen by each instrument at a given time, as can be seen from Fig. 7. In the left plot of the figure, we plotted T2 versus T3 seeing for all measurements taken on 16 September. The right part of the figure shows the comparison of the T2 DIMM measurement with the measurement from the same DIMM taken 3 min later. The scatter in the right part of the figure is significantly larger than that in the left part, thus demonstrating that real changes in turbulence on time scales of minutes cause changes in seeing values much larger than the average difference between the two instruments. Thus, a large part of the scatter in Figs. 5 and 6 is
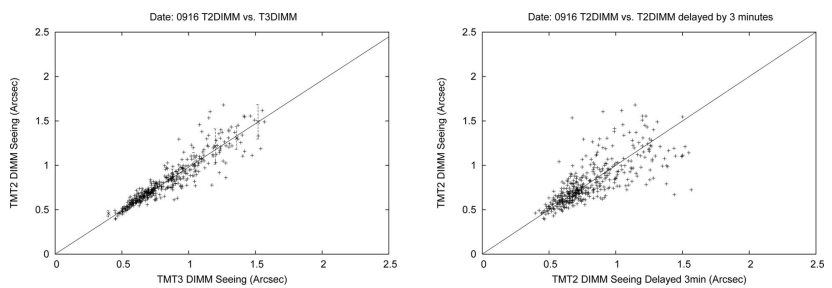

Fig. 7. Data for 16 September: on the left we plot T2 versus T3 DIMM measurements for all data points taken during this night. In the right image we plot the T2 measurement versus the measurement from the same DIMM taken 3 min later. The larger scatter in the right plot indicates that turbulence differences along the respective line of sight at the time of measurement is the dominant cause of the scatter in this and the two previous figures.
Table 3. Dependence of Median Seeing Difference Between T2 and T3 (T2-T3) on Different Seeing-Dependent Strehl Ratio Limits ${ }^{a}$

\begin{tabular}{|c|c|c|c|c|}
\hline$S_{0}$ & $f$ & $N_{\text {points }}$ & $\begin{array}{l}\text { Median Differential } \\
\text { (arc sec) }\end{array}$ & $\begin{array}{l}\text { Mean Differential } \\
(\operatorname{arc~sec})\end{array}$ \\
\hline 0.00 & - & 22,309 & 0.041 & 0.076 \\
\hline 0.10 & 5.99 & 22,309 & 0.041 & 0.076 \\
\hline 0.20 & 5.60 & 22,084 & 0.039 & 0.075 \\
\hline 0.30 & 3.69 & 18,250 & 0.020 & 0.036 \\
\hline 0.33 & 3.00 & 16,829 & 0.017 & 0.032 \\
\hline 0.35 & 2.69 & 15,600 & 0.014 & 0.030 \\
\hline 0.38 & 2.33 & 11,940 & 0.016 & 0.033 \\
\hline 0.40 & 2.14 & 8952 & 0.007 & 0.026 \\
\hline 0.41 & 2.06 & 7013 & -0.002 & 0.013 \\
\hline 0.42 & 1.99 & 4903 & -0.011 & 0.003 \\
\hline 0.43 & 1.92 & 3027 & -0.021 & -0.010 \\
\hline 0.44 & 1.86 & 2247 & -0.025 & -0.014 \\
\hline 0.45 & 1.81 & 1175 & -0.028 & -0.010 \\
\hline
\end{tabular}

${ }^{a}$ The difference is very small for values of $S_{0}$ larger than 0.3 . See text for more explanations.

caused by the fact that start times of individual data acquisitions were not synchronized and that the telescopes look through different air columns in spite of their close proximity. This scatter averages out if data are acquired for a sufficiently long period of time and therefore does not cause a measurement bias.

In addition to using data only from the nights for which we know that both T2 and T3 were well aligned, we also applied the Strehl limit of Eq. (5) to the data of the Tololo T2-T3 comparison campaign using all data, that is, including data points for which the optical alignment of T2 was shown to be bad. The results for different values of $S_{0}$ are shown in Table 3. The case $S_{0}=0.4$ is also shown in Fig. 8 .

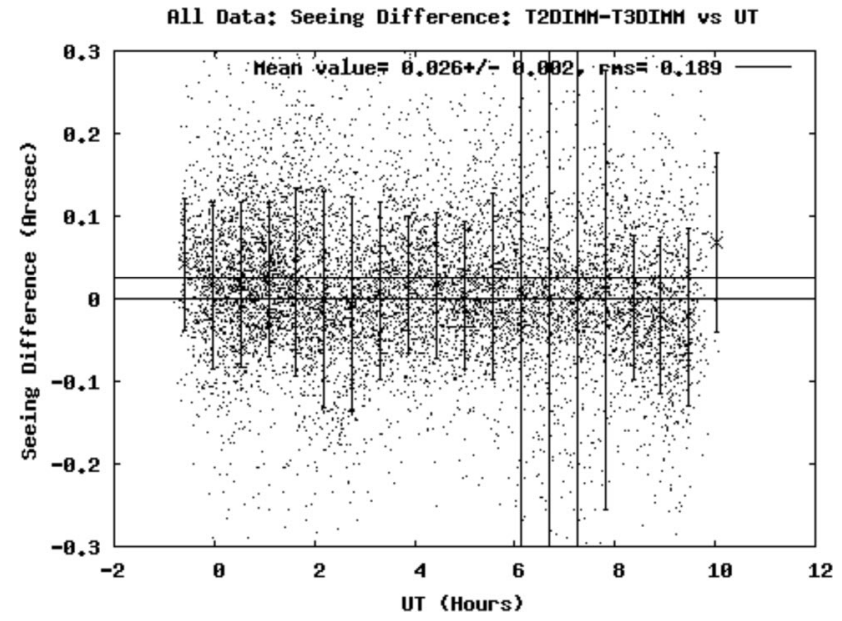

Fig. 8. Difference between the seeing measured by T2 and T3 during the Tololo campaign. All data points, including those for which the optical alignment was known to be bad, were used. Equation (5) with $S_{0}=0.4$ and $f=2.14$ was then applied to identify corrupted data points, which were excluded from the points shown here. The dotted line shows the best fit horizontal line to the data, corresponding to the mean of the data set, while the crosses are the median values for the respective data bins. The error bars indicate the rms scatter in the data bins. 
In the table, the third column contains the numbers of data points remaining after the Strehl limit was applied. (For comparison, the number of data points in region $\mathrm{C}$ in Fig. 3 is $~ 7000$ ). The fourth column is the median of the difference between the T2 and T3 seeing measurement. The last column gives the mean difference of the seeing measurements. We can see that the mean and median differences are generally very small for values of $S_{0}$ larger than 0.3. (In the last rows, so few data points remain that the statistics cannot be considered representative.) For the cases of $S_{0}$ between 0.41 and 0.45 , both the median and mean differences between the measured seeing values are very small. This confirms that our seeing measurements taken with the TMT DIMMs operating under the same conditions are comparable at the level of approximately $0.02^{\prime \prime}$.

Even though the data shown in Figs. 5 and 6 cover approximately one month, with several hundred data points per night, a small but statistically significant difference of 17 mas remains. This difference might be caused by several sources. (1) Even at Strehl ratios above 0.4 , a small bias due to optical aberrations might still be present. (2) Errors in the determination of the configuration parameters (plate scale, aperture sizes, detector noise, etc.) might be sources of systematic errors. (3) There might be real turbulence differences between the T2 and T3 locations at CTIO, especially considering the number of structures on the summit plateau. Investigating which of these effects is the cause of the remaining systematic difference would require a longer comparison campaign, ideally at a site without any structures. We decided that this was not necessary as the achieved repeatability of the measurements is sufficient for our purposes.

We nevertheless have indications that at least part of the difference is caused by local turbulence effects. Figure 9 shows the seeing difference between T2 and T3 as measured on 23 September. While the average difference for the night, $\sim 3$ mas, is very small, a large systematic difference is visible between 2:00 and 4:00 UT. In addition, this difference is negative for the first hour and positive during the next hour. A look at the meteorological conditions during the night shows that the wind, both speed and direction, changed right as these changes occurred (see the right plot of
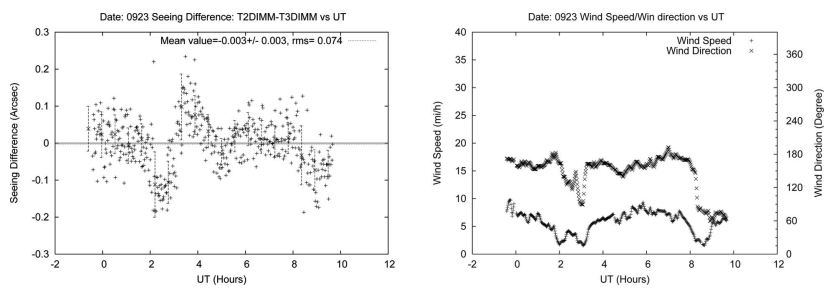

Fig. 9. Data for 23 September: The wind speed and direction (right plot) changed suddenly between 2:00 and 4:00 UT causing large systematic differences between the seeing measured by T2 and T3 (left plot) during this period. The error bars show the standard deviation of seeing difference in respective UT bins. The average difference for the entire night is very small (3 mas).
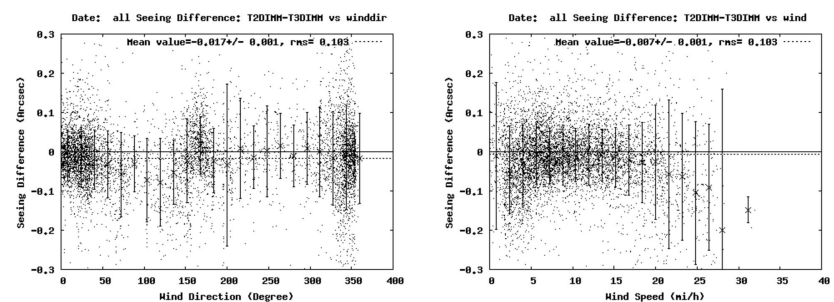

Fig. 10. Left plot: seeing difference (T2-T3) versus wind direction for data from 15 September to 5 October. Right plot: seeing difference versus wind speed for same period. The error bars show the standard deviation of seeing difference in respective bins. The two plots show that the seeing difference between T2 and T3 changes with wind direction and speed and is thus at least partially caused by local turbulence effects.

Fig. 9). This was a night with mostly southern winds (wind direction $180^{\circ}$ ), meaning that the wind flowed past the summit buildings before reaching our DIMMs. The sudden differences between the two DIMMs are therefore almost certainly caused by real differences in local turbulence. Plots of the measured seeing difference versus wind direction and wind speed for all nights from 15 September to 5 October further corroborate this assumption (see Fig. 10). Thus, part of the remaining difference of 17 mas between T2 and T3 is likely caused by local turbulence effects, making this difference an upper limit of the repeatability of our DIMM measurements.

A similar but not as intensive comparison between the TMT DIMMs and the Tololo facility DIMM [6] was also performed. The systematic difference (Tololo DIMM seeing minus TMT DIMM seeing) was found to be of the order of $0.03-0.05$ arc sec. This slightly larger discrepancy than that between T2 and T3 is likely caused by the larger difference in local turbulence conditions (larger separation as well as the fact that the Tololo DIMM is operating behind a halfclosed dome, while the TMT DIMMs operate entirely in the open), by the fact that comparison data exist for a shorter period of time and that slightly different hardware and analysis methods are used. It shows, however, that DIMMs using 2D and 1D image motion data produce results that agree at levels well below 0.1 arc sec if sufficient care is taken. A difference on the same order was found during tests at San Pedro Mártir (SPM) Observatory when comparing the TMT DIMM installed there with another Tololo-style DIMM that we brought to SPM for this purpose.

An equivalent analysis of the overall seeing measured by the TMT MASS units (T2 and T3 both have a MASS unit) shows an agreement of better than 0.05 arc sec on average. Details of the MASS comparison will be reported in a follow-up publication.

\section{Conclusion}

We have shown that the seeing values measured with two TMT DIMMs at Cerro Tololo Inter-American Observatory agree on average to better than 0.02 arc sec. The remaining difference is likely caused at least in part by real differences in turbulence rather than 
instrumental effects. We found that the optical alignment of the telescope-DIMM system affects the data more sensitively than we had previously expected. We established the Strehl ratio of the DIMM data as a metric that can be used to ensure that data are valid and comparable between instruments operating under the same conditions. We have thus confirmed the repeatability of our measurements. Ensuring reproducibility (agreement under different conditions or establishing an absolute calibration) is not possible through a side by side comparison of identical instruments, but only either through a complete bias control mechanism or through comparison between different instruments that are not subject to the same biases. This is beyond the scope of this paper. From the preliminary work we have done in this respect, we believe, however, that the absolute accuracy of our DIMM measurements is similar to the repeatability described above.

The authors thank the staff of the Cerro Tololo Inter-American Observatory for their support and hospitality during our work at CTIO. Special thanks are due to Gale Brehmer and Oscar Saa for their excellent help. We thank Andrei Tokovinin from CTIO for providing his simulation code.
The authors gratefully acknowledge the support of the TMT partner institutions. They are the Association of Canadian Universities for Research in Astronomy (ACURA), the California Institute of Technology, and the University of California. This work was supported as well by the Gordon and Betty Moore Foundation, the Canada Foundation for Innovation, the Ontario Ministry of Research and Innovation, the National Research Council of Canada, and the U.S. National Science Foundation.

\section{References}

1. M. Sarazin and F. Roddier, "The ESO differential image motion monitor," Astron. Astrophys. 227, 294-300 (1990).

2. A. Tokovinin, "From differential image motion to seeing," Publ. Astron. Soc. Pac. 114, 1156-1166 (2002).

3. V. Kornilov, A. A. Tokovinin, O. Vozyakova, A. Zaitsev, N. Shatsky, S. F. Potanin, and M. S. Sarazin, "MASS: a monitor of the vertical turbulence distribution," Proc. SPIE 4839, 837-845 (2003).

4. A. Tokovinin, "Influence of defocus on DIMM," MASS profiler report, available at http://www.ctio.noao.edu/atokovin/profiler/ archive.html (2004).

5. R. J. Noll, "Zernike polynomials and atmospheric turbulence," J. Opt. Soc. Am. 66, 207-211 (1976).

6. These data are available at http://www.ctio.noao.edu/ telescopes/dimm/dimm.html (2002). 